\title{
Les circulaires Marcellin-Fontanet
}

Par Danièle Lochak

In Hommes \& Migrations, 2020/3, « 1973, L'année intense », p. 14-17

Il s'agit de deux circulaires datées, l'une du 24 janvier 1972, émanant du ministre de l'intérieur, l'autre du 23 février 1972, émanant du ministre du travail. Leur célébrité n'est pas usurpée car elles représentent à beaucoup d'égards un moment clé dans l'histoire de l'immigration. Elles annoncent en effet un tournant décisif et durable de la politique migratoire; elles sont à l'origine des premières grandes luttes de sans-papiers ; et leur annulation par le Conseil d'État, trois ans plus tard, démontre l'utilité de l'arme contentieuse pour défendre la cause des immigrés.

\section{Le signe annonciateur de la politique de « maîtrise des flux migratoires »}

Les mesures prises à la Libération témoignaient d'une volonté politique clairement affirmée de contrôler l'immigration et de rompre avec la période de l'entre-deux guerres où on l'avait abandonnée aux fluctuations de l'offre et de la demande et aux initiatives du patronat. L'Office national d'immigration (ONI) se voit donc conférer le monopole du recrutement et de l'introduction en France des travailleurs étrangers, et le droit au séjour est subordonné à la production d'un contrat de travail dûment visé par les services de l'emploi.

Mais très vite l'immigration a échappé au cadre institutionnel prévu. Les besoins de maind'œuvre sont tels que la réglementation n'est guère respectée. En dépit des textes, les travailleurs étrangers entrent en France sous couvert d'un simple passeport de touriste, ou même clandestinement ; ils trouvent sans peine à s'embaucher et obtiennent ensuite aisément les documents carte de séjour et carte de travail - qui régularisent leur situation. Le dispositif législatif, à l'évidence inadapté, reste inappliqué, avec l'assentiment implicite, voire explicite, des pouvoirs publics. On connaît la phrase célèbre d'un ministre des Affaires sociales, prononcée en 1966 : « L'immigration clandestine n'est pas inutile car si l'on s'en tenait à l'application stricte des règlements et accords internationaux, nous manquerions peut-être de main-d'œuvre ».

Cette immigration spontanée, dite aussi «sauvage », encouragée par les pouvoirs publics aussi longtemps qu'elle répond aux besoins immédiats de l'économie française, va commencer à apparaître comme un problème à la fin des années 1960. Jouent ici la transformation de la conjoncture économique, marquée notamment par l'augmentation du chômage, mais aussi les conséquences sociales de cette immigration non contrôlée : logements insalubres, extension des bidonvilles, multiplication des accidents du travail aux conséquences mortelles sont autant d'éléments qui suscitent la dénonciation de plus en plus virulente des « marchands d'hommes » et des «marchands de sommeil $»^{1}$. En 1968, alors que le taux des régularisations atteint $82 \%$ des admissions au séjour, des instructions sont données pour en ralentir le nombre : une circulaire Massenet exclut en principe les régularisations dans les branches d'activité excédentaires. Elle n'est cependant pas appliquée strictement. À son tour le VI e Plan (1971-1975) plaide pour une planification plus rigoureuse de la main-d'œuvre, relevant notamment qu' " un recours à l'immigration étrangère qui demeure relativement important $[\ldots]$ peut être source de déséquilibre en l'absence de politique correctrice » et, plus loin, que, « d'après les prévisions, le solde net migratoire devrait s'élever, pour la période 1970-1975, à environ 80.000 actifs par an, alors qu'il a atteint 110.000 en 1969, 120.000 en 1970. Le Gouvernement, poursuit-il, entend élargir

\footnotetext{
${ }^{1}$ Sammut Carmel, « L'immigration clandestine en France depuis les circulaires Fontanet, Marcellin et Gorse », in Les travailleurs étrangers en Europe occidentale. Actes du Colloque organisé par la Commission nationale pour les études et les recherches interethniques, Paris-Sorbonne, du 5 au 7 juin 1974 - https://www.persee.fr/doc/ierii_1764-8319_1976_act_6_1_921
} 
le champ des procédures régulières de telle sorte que les services chargés du contrôle des introductions de main-d'œuvre étrangère puissent veiller à ce que les introductions ne soient autorisées qu'avec l'octroi au travailleur étranger d'un contrat d'une durée suffisante et comportant des garanties précises en matière de salaires, de conditions de travail et de logement ». Une préconisation que vont mettre en œuvre les circulaires Marcellin-Fontanet ${ }^{2}$. Mais, sous couvert d'un retour à la philosophie de 1945 : ajuster l'accès à l'emploi des immigrés aux besoins du marché du travail français, elles précarisent la situation des travailleurs étrangers dont le droit au séjour est perpétuellement susceptible d'être remis en cause.

Sans entrer dans la description détaillée du contenu des circulaires, on peut en rappeler les principales dispositions.

- Le monopole de l'ONI est réaffirmé pour l'introduction en France de travailleurs immigrés. Il s'accompagne d'une procédure dite de compensation : l'employeur qui souhaite recruter doit préalablement déposer l'offre de travail à l'ANPE afin de s'assurer que l'emploi offert ne peut être pourvu sur le marché du travail français. L'employeur doit par ailleurs remplir une attestation de logement par laquelle il s'engage à procurer au travailleur un logement répondant à certaines conditions (salubrité, densité d'occupation, montant du loyer). Cette obligation imposée à l'employeur, apparemment favorable au travailleur, le met en réalité dans une situation de dépendance accrue par rapport à son patron, puisque, s'il perd son emploi, il perd aussi son logement et son autorisation de séjour.

- Une procédure de régularisation est maintenue, obéissant aux mêmes conditions de fond que la procédure d'introduction (notamment examen préalable de la situation de l'emploi par l'ANPE et attestation de logement) ; mais en sont exclus, sauf cas exceptionnel, les étrangers entrés en France comme "touristes » qui demandent à exercer un emploi de manœuvre ou d'ouvrier spécialisé ou une profession excédentaire en main-d'œuvre.

- La délivrance de la carte de séjour est subordonnée à la production d'un contrat de travail d'une durée d'un an, visé par les services de la main-d'œuvre, et d'une attestation de logement. Le contrat de travail visé vaut autorisation de travail pour l'emploi en cause, pendant la première année, là où précédemment était délivrée une carte de travail qui est valable pour une profession et une région déterminées.

- La durée des titres de séjour et de travail est harmonisée : ils auront désormais la même période de validité et expireront à la même date - ce qui veut dire concrètement que la perte de la carte de travail entraînera celle de la carte de séjour.

Les circulaires Marcellin-Fontanet sont bien annonciatrices d'un tournant de la politique migratoire puisque, deux ans plus tard, les pouvoirs publics décident la suspension officielle de l'immigration de travailleurs.

\section{Les premières luttes de sans-papiers}

Dès qu'elle est connue, la circulaire Fontanet entraîne une très forte mobilisation du monde associatif et syndical ${ }^{3}$. Le 7 février 1972, la CGT et la CFDT lancent une semaine d'information et d'action pour « exiger l'égalité effective des droits entre travailleurs immigrés et français ». L'usine Pennaroya de Lyon se met en grève dès le 9 février, les éboueurs parisiens en décembre. Tout au long de l'année des manifestations rassemblent des milliers de personnes dans plusieurs

\footnotetext{
${ }^{2}$ Sylvain Laurens décrit la genèse de cette conversion du gouvernement à une politique de « maîtrise des flux ». Il montre l'influence convergente des agents de la Direction de la population et des migrations et de ceux du ministère de l'intérieur qui, bien qu'inspirés par des préoccupations différentes, argumentent en faveur d'une limitation des entrées de travailleurs étrangers. Voir Une politisation feutrée. Les hauts fonctionnaires et l'immigration en France (1962-1981), Belin, 2009, p. 178 et s.

${ }^{3}$ Pour un rappel des grandes étapes de ces mobilisations, voir Sylvain Laurens, op. cit., p. 182-183
} 
villes de France, à l'appel de mouvements d'extrême-gauche et/ou de solidarité avec les immigrés.

Avec l'entrée en vigueur des circulaires au mois d'octobre 1972 des dizaines de milliers de travailleurs sont brutalement confrontés à la perte de tout espoir d'être régularisés et au risque d'être expulsés. De nouvelles formes de mobilisation voient alors le jour, cette fois à l'initiative des intéressés eux-mêmes qui, de façon autonome, engagent la lutte pour obtenir leur régularisation. Leur arme principale sera la grève de la faim ${ }^{4}$.

Le 6 novembre 1972, Saïd Bouziri entame, avec trois autres militants immigrés menacés d'expulsion, une grève de la faim ${ }^{5}$. Elle constitue un point de départ pour d'autres actions analogues qui se développent pendant plusieurs mois à travers toute la France, menées majoritairement par des Tunisiens, qui sont les plus touchés, rejoints par des Marocains mais aussi des Mauriciens, des Pakistanais, des Yougoslaves et des Turcs. Un mouvement de soutien à ces grèves s'organise et diversifie ses moyens de lutte : grève des OS de Renault-Billancourt ; occupations des bureaux de la main-d'œuvre ; manifestations, rassemblements et meetings pour réclamer l'abrogation des circulaires.

Face à l'ampleur de la mobilisation, le gouvernement recule. Les circulaires Gorse de l'été 1973 suspendent provisoirement l'exécution de la circulaire Fontanet et accordent un délai pour permettre, sous certaines conditions, à ceux qui sont entrés avant le $1^{\text {er }}$ juin 1973 de demander la régularisation de leur situation. Entre 35000 et 40000 personnes auraient bénéficié de ces mesures transitoires. La lutte des sans-papiers a donc payé, traçant la voie pour les combats à venir.

\section{Une victoire contentieuse tardive mais porteuse d'avenir}

L'action contentieuse elle aussi va être couronnée de succès. Le 13 janvier 1975, par un arrêt Da Silva et CFDT, le Conseil d'État annule plusieurs dispositions des circulaires Marcellin-Fontanet. En réalité, la requête a été rédigée par un des membres fondateurs du Gisti, qui vient de se créer ; mais comme l'association n'est pas encore déclarée, il est décidé de la présenter au nom d'un OS de Renault de nationalité portugaise et de la CFDT6.

Par sa décision du 13 janvier 2015, le Conseil d'État annule trois dispositions importantes des circulaires attaquées :

- l'obligation d'annexer au contrat de travail l'attestation de logement - car cette condition qui n'est prévue par aucun texte législatif ou réglementaire et il n'était donc pas de la compétence des ministres de l'ajouter;

- le remplacement de la carte de travail par le contrat de travail visé pendant la première année de séjour - mesure que le ministre du travail était incompétent pour prendre ;

- enfin et surtout l'interdiction faite aux préfets de régulariser la situation des travailleurs entrés en France en dehors de la procédure d'introduction prévue par les textes.

La décision intervient trop tard pour produire tous ses effets puisque dans l'intervalle est intervenue la suspension de l'immigration de travail qui marque le début - cette fois officiel d'une nouvelle phase de la politique d'immigration.

Son impact ne saurait pour autant être sous-estimé, à la fois sur le plan juridique et sur le plan politique.

\footnotetext{
${ }^{4}$ Sur le recours aux grèves de la faim et sa place dans le répertoire d'action des sans-papiers, voir Johanna Siméant, La cause des sans-papiers, Presses de Science Po, 1998, p. 280 et s.

5 « De la clandestinité à la reconnaissance», Interview de Saïd Bouziri et Driss El Yazami , Plein droit, juin 1990.

${ }^{6}$ Le rédacteur de la requête était Philippe Waquet, avocat au Conseil d'État et à la Cour de cassation, qui livre une analyse la portée juridique mais aussi politique de cette décision : Philippe Waquet, « Retour sur deux combats pour le droit des étrangers », Défendre la cause des étrangers en justice, Dalloz-Gisti, 2009, p. 15.
} 
Sur le plan juridique, l'arrêt consacre l'existence d'un pouvoir de régularisation, présenté comme une attribution fondamentale de l'administration : les circulaires sont annulées « en tant qu'elles ont étendu l'impossibilité de demander une autorisation de travail pour certaines professions aux étrangers entrés comme touristes ». Le ministre ne pouvait pas, par une simple circulaire, supprimer ou limiter ce pouvoir.

La portée du principe ainsi posé est capitale. C'est lui qui permet aujourd'hui encore d'obtenir, par des mobilisations syndicales ou associatives, la régularisation des sans-papiers. En août 1996 encore, dans le contexte de l'évacuation de l'Église Saint-Bernard, le Conseil d'État a réaffirmé que l'autorité administrative avait le pouvoir de procéder à de telles régularisations et qu'elle pouvait prendre à titre exceptionnel une mesure gracieuse favorable à l'intéressé, justifiée par sa situation particulière - ajoutant que tout demandeur de régularisation a le droit de voir son cas examiné.

Sur le plan politique, la décision, dont la presse se fait largement l'écho, constitue incontestablement une victoire contre le gouvernement. Elle démontre aussi, y compris à des militants a priori méfiants à l'égard du droit, qu'ils appréhendent avant tout comme l'instrument de la classe dominante, que l'arme contentieuse peut être utile pour défendre la cause des immigrés. Cette utilité sera confirmée trois ans plus tard, de façon encore plus éclatante, par l'arrêt Gisti du 8 décembre 1978 annulant le décret qui subordonnait le regroupement familial à l'engagement des membres de famille de ne pas travailler. 